\title{
Sequential Logic Optimization with Implicit Retiming and Resynthesis*
}

\author{
S. Bommu, M. Ciesielski, N. O'Neill, P. Kalla \\ University of Massachusetts \\ Department of Electrical \& Computer Engineering \\ Amherst, MA 01003, USA \\ Tel: (413)-545-0401 Fax: (413)-545-1993. \\ email: ciesiel@ecs.umass.edu
}

\begin{abstract}
This paper* introduces a new logic transformation that integrates retiming with algebraic and Boolean transformations at the technology-independent level. It offers an additional degree of freedom in sequential network optimization resulting from implicit retiming across logic blocks and fanout stems. The application of this transformation to sequential network synthesis results in the optimization of logic across register boundaries. We have implemented our new technique within the SIS framework and demonstrated its effectiveness in terms of cycle-time minimization on a set of sequential benchmark circuits.
\end{abstract}

\section{Keywords}

Sequential Logic Synthesis, Logic Optimization, Retiming

\section{INTRODUCTION}

Over the years, sequential circuit synthesis has been a subject of intensive investigation. Though synthesis of combinational logic has attained a significant level of maturity, sequential circuit synthesis is lagging behind. In current state of affairs, sequential networks are first optimized by applying combinational network transformations to the logic between the register boundaries, and mapped into the gate-level network. The resulting network is then often optimized by applying retiming transformation [8].

Retiming is the process of relocating the registers across logic gates without affecting the underlying combinational logic structure. It can be used to minimize cycle-time or the number of registers under the cycle-time constraint.

* This work has been supported in part by a grant from NSF under contract No. MIP9613864, and in part by the Healey Endowment Grant from the University of Massachusetts.

S. Bommu is now with NEC C\&C Research Labs, Princeton, N.J. 
While in principle retiming can be applied at various levels of synchronous system design, it has been traditionally used as a structural transformation in gate-level circuit optimization. As such, gate-level retiming exploits only one degree of freedom in circuit optimization, namely, the relocation of registers. Furthermore, gate-level retiming does not take into account the prospective logic simplification. Potential for the optimization by subsequent re-synthesis is very limited, as it is typically applied to the logic between register boundaries.

In this paper we investigate the issue of retiming at the technology independent level. We introduce a novel and efficient approach to synthesis and optimization of synchronous sequential circuits, in which retiming is performed implicitly during logic optimization.

There have been several attempts to combine retiming with algebraic network transformations in the quest to optimize the logic across register boundaries. Peripheral retiming introduced by Malik et. al. [10] optimizes the underlying combinational logic after a temporary relocation of registers to the periphery of the circuit. It suffers from a limited mobility of registers during the peripheral movement phase. DeMicheli [2] introduced a concept of synchronous divisors and used it in local logic optimization across the register boundaries. Lin [9] formalized the theory for synchronous extraction to detect potential common divisors. Both methods operate on the structural specification of a synchronous circuit, and do not take into account the prospective logic simplification during synchronous division. Dey et al [3] proposed a method to improve effectiveness of retiming by attempting to eliminate retiming bottlenecks. Chakradhar et al. [1] introduced special timing constraints which are used to resynthesize the circuit. The modified circuit is subsequently retimed, and the constraints (if satisfied by the delay optimizer) guarantee that the circuit is retimable and meets the desired cycle time.

Retiming has been also used in the context of minimizing latency (rather than clock period) in pipelined circuits. A number of papers addressed a problem of combining retiming with architectural and structural transformations to minimize the latency and/or throughput. The scheme proposed by Potkonjak et al. [11] uses retiming to enable algebraic transformations that can further improve latency/throughput. Hassoun et al [5] introduced a concept of architectural retiming which attempts to increase the number of registers on a latency-constrained path, without increasing the overall latency. These seemingly contradicting goals are achieved by implementing "negative" registers using precomputation and prediction techniques. In the process, the circuit is structurally modified to preserve its functionality.

Most of the techniques mentioned above operate on a structural representation of the network. The cost function that guides retiming in network optimization does not take into account the potential for subsequent logic simplification. In contrast, our approach takes into account the effect of retiming on logic simplification. It operates directly on a functional specification 
given in terms of synchronous Boolean expressions. It is an iterative synthesis process which integrates retiming with extraction, collapsing, and node simplification, into one synchronous transformation. It efficiently handles retiming across fanout stems while preserving initial state. It also provides a simple method to compute initial state of the resynthesized circuit, consistent with the original network specification.

\section{PRELIMINARIES}

A Boolean function, $F$, of $n$ variables is a mapping $f: B^{n} \longrightarrow B$, where $B=\{0,1\}$. A literal is a Boolean variable or its complement. A cube is defined as a product of literals. The support of a Boolean function is defined as a set of all variables that appear in the function. An expression is said to be cube-free when it cannot be factored by a cube. A kernel of an expression is a cube-free quotient of the expression divided by a cube. Extraction is the process of factoring out a subexpression from one or more logic functions of a network and creating a new node for the extracted expression. Collapsing or elimination is the process of (re)expressing a Boolean function representing a node in the logic network in terms of the support variables of its fanin node.

Forward retiming is the operation of shifting the registers from the inputs to the outputs of a node in a Boolean network; backward retiming is the reverse operation. A node in the network can represent an arbitrary Boolean function. It has been shown that such a transformation preserves the behavior of the circuit [8]. Forward and backward retiming transformations are illustrated in Fig. 1 a). A node is said to be forward (backward) retimable if each of its input (output) edges contain a register. Retiming across a fanout stem is the operation of forward retiming of a multiple-fanout register across its fanout stem. Retiming across a fanout stem imposes an equivalence relation on the fanout registers. All network transformations and initial state computation, must take into account this register equivalence. An expression is called a retimable expression if all the variables in its support set are register variables.
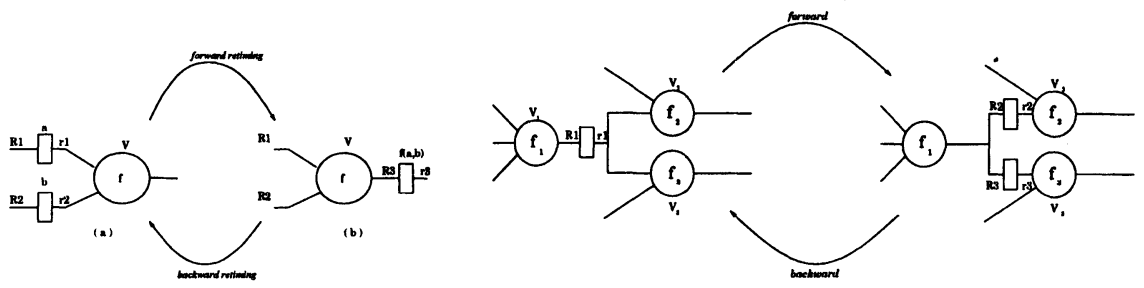

Figure 1 a) Retiming of a logic node b) Retiming across a fanout stem

Associated with each register is a pair of variables $\left(R_{i}, r_{i}\right)$, where $R_{i}$ is the input to the register and $r_{i}$ is its output, referred to as a register variable. 
The variables $r_{i}$ and $R_{i}$ can be viewed as inputs and outputs, respectively, of the combinational part of the sequential network, with registers providing feedback paths.

\section{THEORY AND ALGORITHMS}

Traditional retiming across a logic gate in a gate-level network (or across a node in a Boolean network) can be extended to a retiming across an arbitrary subexpression of the original logic function. Such a retiming, combined with the extraction of a suitable expression, forms the basis of our new sequential transformation. We shall refer to it as the logic retiming transformation, for lack of a better term. The following sections describe the operations involved in logic retiming.

\subsection{Retime Extraction}

Example 1: Consider the sequential logic network represented by the following equations and shown in Fig. 2a):

$O_{1}=i_{2}+r_{3} i_{1}+r_{1} r_{2} i_{1}$

$R_{1}=r_{1} r_{2} i_{2}+r_{3} i_{2}$

$R_{2}=i_{1} r_{2}$

$R_{3}=i_{2}+i_{1} r_{3}$

In these equations $i_{i}$ denotes a primary input and $r_{i}$ denotes a register variable (present state variable). $O_{i}$ is a primary output function and $R_{i}$ is a register function (next state function).
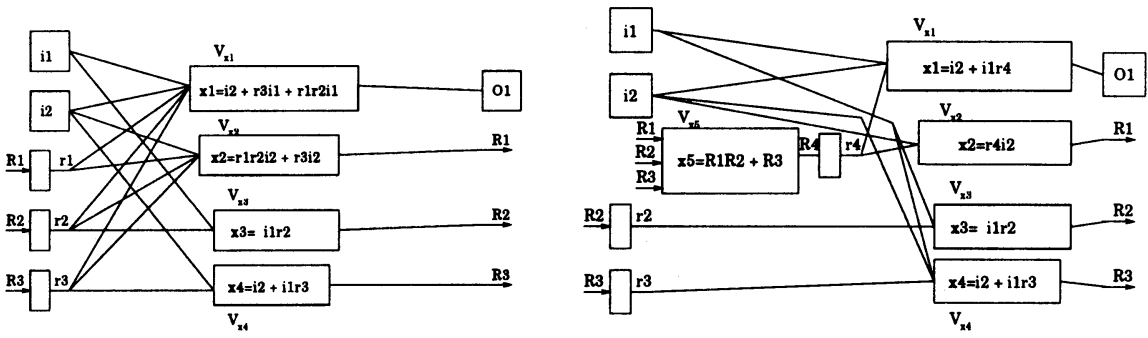

Figure 2 a) The original network, b) Retime-extraction

Consider subexpression $k_{r}=r_{1} r_{2}+r_{3}$, common to $O_{1}$ and $R_{1}$. This subexpression can be extracted from the expressions for $O_{1}$ and $R_{1}$ and used to create a new node in the network, $V_{x 5}$. Since all the inputs to $k_{r}$ are register 
variables, this expression is forward retimable. Forward retiming across $V_{x 5}$ leads to the creation of a new register represented by variables $\left(R_{4}, r_{4}\right)$. After the retiming, the expression for $R_{4}$ is then given in terms of register input variables $R_{i}$, as illustrated in Fig. 2 b).

This transformation can be expressed as a new operation, called retimeextraction, which is the basis of our logic retiming transformation. For a given retimable expression $k_{r}$, the following steps implement retime-extraction:

1. For every node $f_{i}$ of the network, containing expression $k_{r}$, substitute the expression with a variable $r_{k}$.

2. Introduce a new node corresponding to $k_{r}$ expressed in terms of register input variables, $R_{i}$. Represent it by register function $R_{k}$.

3. Introduce a new register $\left(R_{k}, r_{k}\right)$.

It should be emphasized that, whenever the register variables in the support of retimable expression $k_{r}$ fan out to other functions, the retime-extract operation involves implicit retiming across fanout stems. In our example this applies to registers $R_{2}, R_{3}$ which have multiple fanouts. Consequently, a set of equivalence relations will be imposed on these registers and used in the subsequent logic simplification. On the other hand, if a register involved in the retime-extraction fans out only to the retimable expression, it will be rendered redundant by the transformation and subsequently removed. In our example, $R_{1}$ fans out only to the retime-extracted expression, and can be removed along with the associated logic function (Fig. 4).

\subsection{Collapsing and Simplification}

The next step is to collapse the node represented by a new variable $R_{k}$ into its fanin nodes, as shown in Fig. 3. The resulting expression is then simplified. Notice the implicit duplication of logic, necessary to perform the collapsing and simplification. This ensures that the functionality of the rest of the network remains unchanged. In our case, logic for $R_{1}, R_{2}, R_{3}$ is duplicated (see the area marked by the dotted line). The simplification is possible, in effect, due to register equivalence imposed on fanout registers. For simplicity, in all the figures, we use the same variable name for each of the registers obtained after retiming across a fanout.

In our case the collapsing and simplification lead to the following expression:

$$
R_{4}=R_{1} R_{2}+R_{3}=\left(r_{4} i_{2}\right)\left(i_{1} r_{2}\right)+\left(i_{2}+i_{1} r_{3}\right)=i_{2}+i_{1} r_{3}
$$

The simplified Boolean expression for $R_{k}$ is also referred to as a retimeexpression $R E\left(k_{r}\right)$. It can be calculated for every retimable kernel or cube $k_{r}$ using the above procedure. The computation of $R E\left(k_{r}\right)$ is central to the logic 


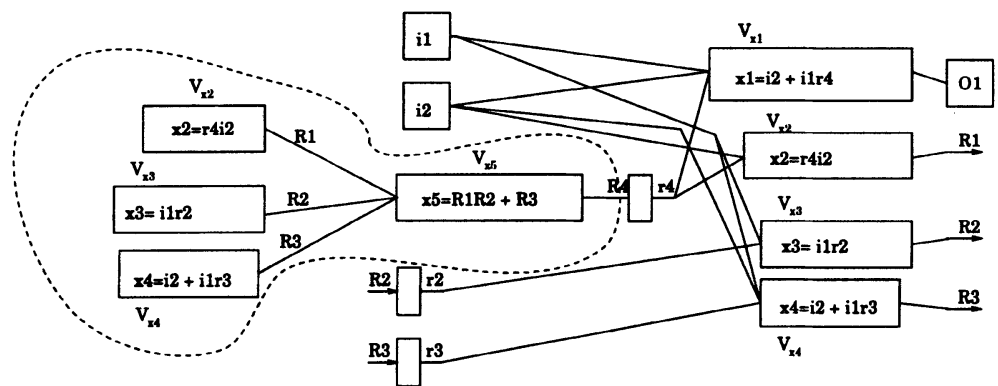

Figure 3 Collapsing of $R_{4}$ into its fanin nodes

retiming transformation. In our example, the expressions associated with $V_{x 5}$ and $V_{x 4}$ are the same $\left(i_{2}+i_{1} r_{3}\right)$ and hence $V_{x 5}$ can be removed, as shown in Fig. 4(a). Finally, notice that register function $R_{1}$ is not used. This is because the register disappeared as a result of retime extraction across $r_{1} r_{2}+r_{3}$. Therefore, the combinational logic function associated with the register function can be deleted. The resulting network is shown in Fig. 4(b). Furthermore, since the register functions $R_{3}, R_{4}$ are identical, the two registers could be merged into one, provided that their initial states are identical, i.e., $r_{3}^{0}=r_{4}^{0}$. Whether this is possible or not, depends on the initial conditions imposed on the network (see the next section on initial state computation).
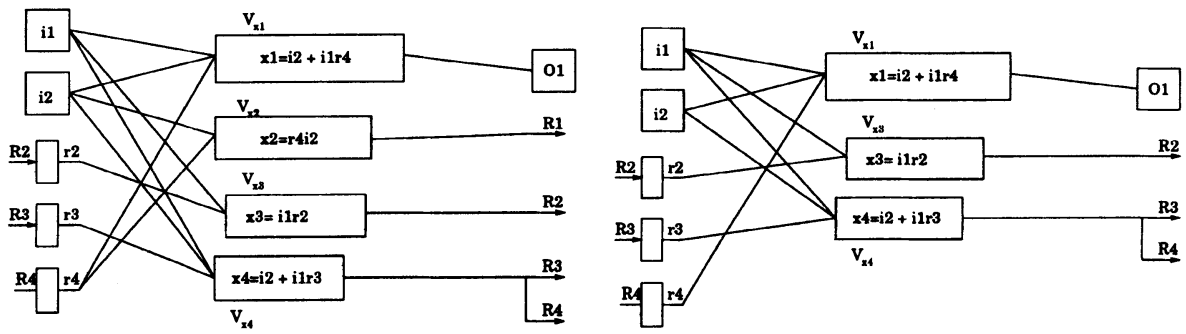

Figure 4 a) Network after simplification b) Final network with redundant logic removed

This network is the direct result of our logic retiming transformation. The retime-extraction, collapsing and simplification transformations are performed implicitly through the computation of the retime-expression.

\subsection{Initial State Computation}

The initial state computation upon forward retiming across an arbitrary logic expression, as formally given in [12], is straightforward. Let $r_{i}^{0}$ be the initial value of a register $\left(R_{i}, r_{i}\right)$. For a retimable expression $k_{r}\left(r_{1}, r_{2}, \ldots, r_{n}\right)$, the 
initial value of the register $\left(R_{k}, r_{k}\right)$, added by the retime-extraction, is given by $r_{k}^{0}=k_{r}\left(r_{1}^{0}, r_{2}^{0}, \ldots, r_{n}^{0}\right)$. For the example above, with retimable expression $k_{r}=r_{1} r_{2}+r_{3}$, the initial value of register $\left(R_{4}, r_{4}\right)$ is then given by $r_{4}^{0}=$ $r_{1}^{0} r_{2}^{0}+r_{3}^{0}$.

\subsection{Cost Modeling}

Logic retiming is characterized by several important properties, which can be illustrated conceptually in Fig. 5. First, it can be shown that logic retiming does not degrade the overall cycle-time under the unit-delay model. Since the retime-expression node $R E\left(k_{r}\right)$ is obtained by means of collapsing and simplification, it will always be appended to the network at the same (last) level as the nodes that are collapsed into it. By definition, the arrival time at the output of this node will be no greater than the latest arrival time in the rest of the network. Hence adding a retime-expression node will not increase the topological longest path under this model.

Realistically, since retime-extraction may increase fanout on some of the nodes (for example node $i_{1}$ in Fig. 4), the critical path delay could actually increase. This may happen, for example, when a node on a critical path fans out to the newly created node, $R E\left(k_{r}\right)$ (see node $V_{1}$ in the figure). This problem can be identified by considering an augmented delay model which takes the fanout factor into consideration.
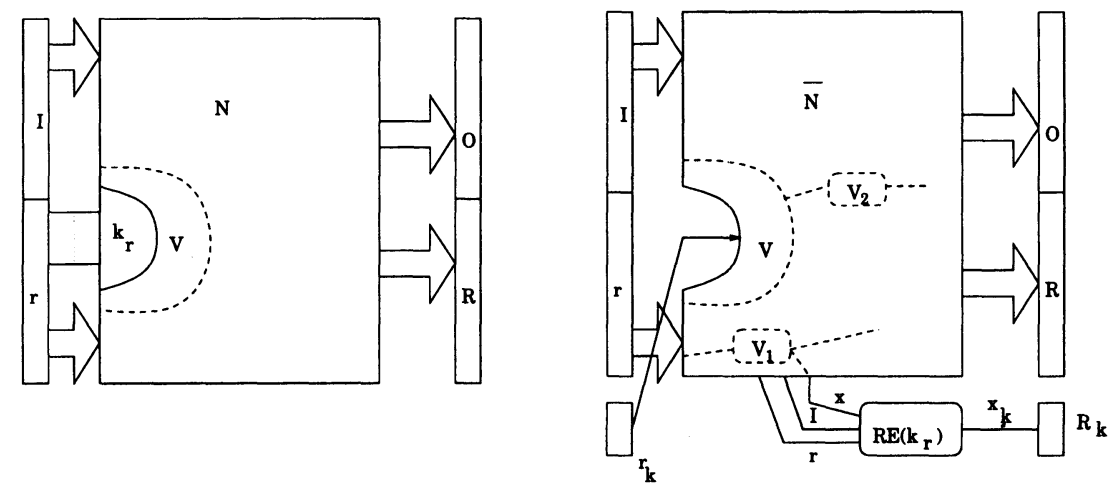

Figure 5 Conceptual view of logic retiming

Finally, observe that the complexity of a node (measured e.g. in the number of literals) that is affected by retime-extraction will always be reduced by the extraction of the retimable expression (see node $V$ in the figure). Since it can be argued that the complexity of a node reflects to a certain degree its delay, the delay of the critical path will be reduced, provided that retime-extraction 
targets the expressions along that path (for example, if $V_{2}$ is on a critical path) and that the fanout increase does not offset the gain due to extraction.

The key element to the efficiency of logic retiming is accurate estimation of the cost associated with a given retimable expression. Fig. 6 illustrates the idea of cost estimation based on simple literal count. It is important to note that the two candidate nodes, $k_{r}$ and $R E\left(k_{r}\right)$, are not yet part of the network. Two gains are computed: $\Delta x$ for standard extraction, and $\Delta r$ for retime-extraction.

$$
\begin{aligned}
\Delta x & =\max (\text { lit_count }(V 1), \text { lit_count }(V 2), \text { lit_count }(V 3)) \\
\Delta r & =\text { lit_count }\left(R E\left(K_{r}\right)\right)
\end{aligned}
$$

The literal counts of nodes $V 1, V 2, V 3$ are computed before extraction or retime-extraction; these include the literals of $k_{r}$. Retime-extraction (which results in the addition of node $\left.R E\left(k_{r}\right)\right)$ is performed if $\Delta r<\Delta x$.

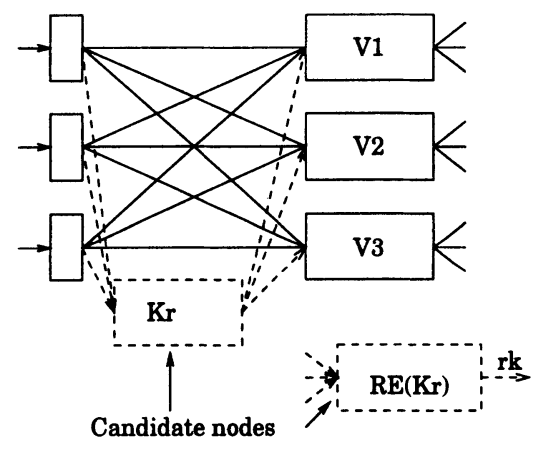

Figure 6 Delay gain estimation based on literal count

Also note that while this approach emphasizes the delay, it can also be used to target the logic area (approximated by the total number of literals). The gain in area can be computed by comparing $\Delta r$ with $\Delta y=\operatorname{lit}$ _count $\left(k_{r}\right)$. Depending on the depth of collapsing and the amount of logic simplification, $\Delta r$ may be greater or smaller than $\Delta y$.

The initial experiments have shown that even this simplistic gain metric can result in cycle-time reduction. A more accurate approach, currently being considered, involves a fast node decomposition using Time-Driven Cofactoring (TDC) [4].

\subsection{Logic Retiming Algorithm}

Logic retiming is an iterative operation comprised of the following steps: 
1. Select a set of candidate subexpressions to be extracted.

2. For each candidate subexpression, check if it is retimable.

3 . If retimable, estimate the delay gain of retime-extraction $(\Delta r)$ and regular extraction $(\Delta x)$. It should be emphasized that the gain $\Delta r$ for the retime-expression $k_{r}$ is based on all the transformations involved: retimeextraction, collapsing and simplification.

4. If retime-extraction gives better gain, perform retime-extraction. Otherwise, perform regular extraction.

\subsection{Comparison with Extraction and Gate-level Retiming}

The following example illustrates that logic retiming can lead to circuit optimization (both in terms of delay and area) that is not possible with conventional multi-level synthesis or gate-level retiming alone, see Fig. 7.

Example 2: Consider again logic network for Example 1 (1). Recall that retime-extraction of expression $k_{r}=\left(r_{1} r_{2}+r_{3}\right)$ resulted in a new variable $R_{4}=R_{1} R_{2}+R_{3}=\left(r_{4} i_{2}\right)\left(i_{1} r_{2}\right)+\left(i_{2}+i_{1} r_{3}\right)=i_{2}+i_{1} r_{3}$

\section{Extraction:}

extract $k_{r}$, introduce variable $x_{5}=r_{1} r_{2}+r_{3}$

$$
\begin{aligned}
O_{1} & =x_{5} i_{1}+i_{2} \\
R_{1} & =x_{1} i_{2} \\
R_{2} & =i_{1} r_{2} \\
R_{3} & =i_{2}+i_{1} r_{3} \\
x_{1} & =\left(r_{1} r_{2}+r_{3}\right)
\end{aligned}
$$

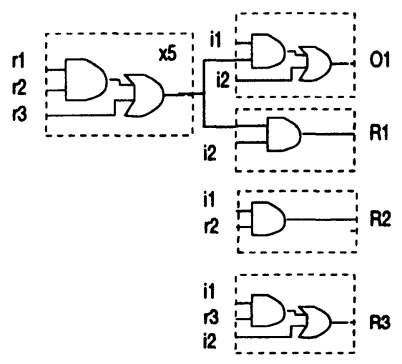

\section{Logic Retiming:} retime_extract across $R_{4}=R_{1} R_{2}+R_{3}$, collapse and simplify.

$$
\begin{aligned}
& O_{1}=r_{4} i_{1}+i_{2} \\
& R_{2}=i_{1} r_{2} \\
& R_{3}=i_{2}+i_{1} r_{3} \\
& R_{4}=i_{2}+i_{1} r_{3}=R_{3}
\end{aligned}
$$

\section{Retiming:}

structural operation, retime across $\left(r_{1} r_{2}\right)$

$$
\begin{aligned}
& O_{1}=\left(r_{5}+r_{3}\right) i_{1}+i_{2} \\
& R_{2}=i_{1} r_{2} \\
& R_{3}=i_{2}+i_{1} r_{3} \\
& R_{5}=R_{1} R_{2}
\end{aligned}
$$

Figure 7 Comparison of logic retiming with extraction and retiming (feedback loops $R_{i} \rightarrow r_{i}$ are omitted for simplicity). 


\section{IMPLEMENTATION AND EXPERIMENTAL RESULTS}

We implemented the logic retiming transformation within the SIS framework. The implementation of the logic retiming algorithm involves the generation of common subexpressions of the combinational part of the network; these common subexpressions are generated using the rectangle intersection algorithm used in SIS. Only those kernels whose value exceeds the user-defined threshold are selected. For each of the selected kernels we compare the regular extraction value with the retime-extraction value using the gain estimation technique.

The cost function, used in our preliminary experiments, is the number of literals in the SOP form, as discussed in section 3.4. Although simplistic, this cost function allowed us to quickly validate the theory. This version of logic retiming yielded delay improvements over the regular extraction transformation. Research is now focused on the application of the concept of retime-extraction to the transformations used in script.delay and other delay optimization techniques such as speed_up. We are also investigating the application of logic retiming to area minimization under cycle-time constraints.

We have tested our technique on a number of sequential circuits from the ISCAS'91 benchmark set. The circuits were input as logic networks in blif format, its local functions (nodes) were collapsed into SOP form. Each circuit was then optimized using logic retiming and independently synthesized with standard SIS multi-level optimization. The circuits were resynthesized and mapped into the standard SIS lib2.genlib library. The script used for logic retiming is identical to the script with conventional SIS transformations, except that the $g k x$ command has been replaced by the "retime kernel extract" ( $r k x)$ command of logic retiming. The general structure of the scripts used in our experiments is given below:

$\frac{\text { script.rkx }}{\text { sweep }}$
collapse or eliminate <threshold>
simplify
$r k x<$ options>
resub - $a$
sweep
simplify

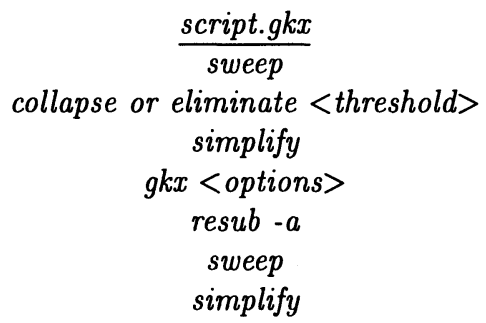

The results are reported in Table 1, which compares the clock-cycle delay, number of registers, and area overhead of the circuits obtained by the two flows. The delay was computed using the mapped delay model. Those circuits which did not contain any retimable kernels are not shown in the table.

Even though our initial implementation of logic retiming used a simplistic 
Table $1 g k x$ vs $r k x$ : Comparison of mapped circuits

\begin{tabular}{cccccccccc}
\hline Ckt & \multicolumn{2}{c}{ rkx } & \multicolumn{3}{c}{ gkx } & \multicolumn{3}{c}{ \% change } \\
\hline & Area & Clk & Reg & Area & Clk & Reg & Area & Clk & Reg \\
\hline s298 & 167040 & 9.59 & 25 & 145232 & 10.95 & 14 & 15 & -12 & 79 \\
\hline $\mathrm{s} 344$ & 198592 & 13.50 & 17 & 187456 & 17.06 & 15 & 6 & -21 & 13 \\
\hline $\mathrm{s} 444$ & 223648 & 9.51 & 24 & 203232 & 13.09 & 21 & 10 & -27 & 14 \\
\hline $\mathrm{s} 526$ & 228288 & 10.10 & 25 & 208336 & 13.64 & 21 & 10 & -26 & 19 \\
\hline $\mathrm{s} 400$ & 266800 & 11.05 & 28 & 211120 & 12.95 & 21 & 26 & -15 & 33 \\
\hline $\mathrm{s} 9234$ & 1156752 & 31.90 & 147 & 1101536 & 38.28 & 135 & 5 & -17 & 9 \\
\hline $\mathrm{s} 5378$ & 1316832 & 25.29 & 189 & 1286672 & 26.31 & 162 & 2 & -4 & 17 \\
\hline $\mathrm{s} 510$ & 245920 & 24.49 & 8 & 223184 & 28.20 & 6 & 10 & -13 & 33 \\
\hline $\mathrm{s} 15850$ & 3912912 & 104.1 & 538 & 3802480 & 108.23 & 504 & 3 & -4 & 7 \\
\hline $\mathrm{s} 1488$ & 629648 & 42.72 & 13 & 607840 & 39.67 & 6 & 4 & 7 & 117 \\
\hline $\mathrm{s} 382$ & 329904 & 10.63 & 33 & 215760 & 13.82 & 21 & 53 & -23 & 57 \\
\hline & & & & & & & & &
\end{tabular}

figure of merit based on literal count, most of the circuits synthesized with this technique showed a significant reduction in delay. We expect that with a better estimation scheme and more selective retime-extraction the transformation will give better and consistent improvement in delay. The algorithm and library used for mapping would also have a significant impact on the delay of the optimized circuit; applying retime-extraction with explicit knowledge of the above information could improve the effectiveness of logic retiming.

\section{REFERENCES}

[1] S.T. Chakradhar, S. Dey, M. Potkonjak, S.G. Rothweiler, "Sequential Circuit Delay Optimization using Global Path Delays", Proc. 1993 Design Automation Conf., 1993, pp. 483-489.

[2] G. De Micheli, "Synchronous Logic Synthesis: Algorithms for CycleTime Optimization", IEEE Trans. on CAD, Vol.10, No.1, Jan. 1991, pp. 63-73.

[3] S. Dey, M. Potkonjak, S. Rothweiler, "Performance Optimization of Sequential Circuits by Eliminating Retiming Bottlenecks", Intl. Conference on $C A D, 1992$, pp. 504-509.

[4] P. Gutwin, P. McGeer, R. Brayton, "Delay Prediction for TechnologyIndependent Logic Equations", Proc. IEEE Intl. Conference on Computer Design, 1992, pp. 468-471

[5] S. Hassoun, C. Ebeling, "Architectural Retiming: Pipelining LatencyConstrained Circuits", Proc. 1996 Design Automation Conf., 1996.

[6] G.D. Hachtel, M. Hermida, A. Pardo, M. Poncino, F. Somenzi, "Re- 
encoding Sequential Circuits to Reduce Power Dissipation", Intl. Conference on $C A D, 1994$, pp. 70-73.

[7] S. Iman and M. Pedram "Logic Extraction and Factorization for Low Power", Proc. 1995 Design Automation Conf., 1995, pp. 248-253.

[8] C. Leiserson, F. Rose, J. Saxe, "Optimizing Synchronous Circuitry by Retiming, Third Caltech Conference on VLSI, 1983, pp. 87-116.

[9] B. Lin, "Restructuring of Synchronous Logic Circuits", Proc. European Design Automation Conference, 1993, pp. 205-209.

[10] S. Malik, E. Sentovich, R. Brayton, A. Sangiovanni-Vincentelli, "Retiming and Resynthesis: Optimizing Sequential Networks with Combinational Techniques", IEEE Trans. on CAD, Vol.10, No.1, Jan. 1991, pp. 74-84.

[11] M. Potkonjak, S. Dey, Z. Iqbal, A. Parker. "High Performance Embedded System Optimization Using Algebraic and Generalized Retiming Techniques", Proc. IEEE Intl. Conference on Computer Design, 1993, pp.498-504.

[12] H.J. Touati and R.K. Brayton, "Computing the Initial States of Retimed Circuits", IEEE Trans. on CAD, (12)1, Jan. 1993, pp. 157-162.

[13] C.Y. Tsui, M. Pedram, C-A Chen, and A. Despain, "Technology Decomposition and Mapping Targetting Low Power Dissipation", Proc. 1994 Conference on CAD, 1994, pp. 82-87.

\section{BIOGRAPHY}

Surendra Bommu received his B.Tech degree in Electrical and Electronics Engineering from Indian Institute of Technology, Madras, India in 1993. He received his M.S. degree from the department of Electrical and Computer Engineering, University of Massachusetts, Amherst in 1996. He is currently working as a research associate at C\&C Research Labs, NEC USA Inc., Princeton. His research interests include Logic Synthesis, High Level Synthesis, and Hardware/Software Codesign.

Maciej Ciesielski received his M.S. in Electrical Engineering from Warsaw Technical University, Poland, in 1974, and Ph.D. in Electrical Engineering from the University of Rochester in 1983. From 1983 to 1986 he was a Senior Member of Technical Staff at GTE Laboratories. Currently he is Associate Professor in the Department of Electrical and Computer Engineering at the University of Massachusetts, Amherst. His research interests include: CAD for VLSI systems, architectural, logic and physical synthesis and performance optimization of IC's. He has served on the technical program committees for ICCAD, ICCD, IWLS, IFIP/IWLAS, and others. He is a member of the IEEE.

Niall O'Neill and Priyank Kalla are graduate students at the University of Massachusetts, Department of Electrical \& Computer Engineering. 